$$
\begin{aligned}
& \sum w_{\sigma}^{2}=\frac{n}{2}\left(1-\varrho_{1}\right) \\
& \sum \sigma r_{1 \sigma}{ }^{2}==\sum \sigma s_{1 \sigma}{ }^{2}=\frac{n}{4} b^{2} \sin ^{2} \varphi\left(1-3 \varrho_{1}+4 \varrho_{2}\right) \\
& \sum \sigma r_{2 \sigma}{ }^{2}=\sum \sigma s_{2 \sigma^{2}}=\left(\sum \sigma r_{2 \sigma}\right)^{2}=\left(\sum \sigma s_{2 \sigma}\right)^{2}=\frac{n}{16} b^{2} \sin ^{2} \varphi\left(1+18 \rho_{1}-19 \rho_{2}\right) \\
& \left(1-3 \varphi_{1}\right) \mu^{4}+8 \varrho_{1} \mu-\left(1+\varrho_{1}\right)=0 \\
& \cos ^{2} d \cdot m(a)^{2}=m(d)^{2}=\frac{4\left(1-\mu^{16}\right)\left(1-3 \varrho_{1}+4 \varrho_{2}\right)+\left(3-4 \mu^{4}+\mu^{16}\right)\left(1+18 \varrho_{1}-19 \varrho_{2}\right)}{3^{2} n\left(1-3 \varrho_{1}\right)^{2}}
\end{aligned}
$$

Setzt man die oben angegebenen Werte von $\rho_{1}$ und $\rho_{2}$ ein, so findet man die einzige positive Wurzel

$$
\mu=0.7266 \text {. }
$$

Diesem Werte entspricht

$$
m=0.7992=45^{\circ} 79 .
$$

Der wahrscheinliche Fehler eines einzelnen Wertes $\Delta \omega_{\sigma}$ ist also 30:89. Schließlich wird

$$
\begin{aligned}
& \cos d \cdot m(a)=m(d)=\frac{63^{\circ} \cdot 34}{\sqrt{n}} \\
& \cos d \cdot \varepsilon(a)=\varepsilon(d)=\frac{42^{\circ} \cdot 7 \pi}{\sqrt{n}}
\end{aligned}
$$

Sternwarte Kiel, 1904 November.

Nachschrift vom 23. Februar 1905, Herro Bohlins Artikel in der soeben erschienenen Nr. 3997 dieser Zeitschrift veranlaßt mich, daran zu erinnern, daß Herr Kobold die Bedingung, daß $\sum_{\sigma} v_{\sigma}^{2}$ ein Minimum werde, für die Bestimmung auch des Radianten eines Sternschnuppenschwarmes benutzt hat (vergl. Astr. Nachr. 3608, Bd. I 5 I, I900, S. 120 ). Herro Bohlins Lösung entspricht dem eindeutigen Problem, den Ausdruck $\sum \sigma \pi_{\sigma} v_{\sigma}^{2}=\frac{1}{x_{3}{ }^{2}} \sum \sigma \frac{v_{\sigma}{ }^{2}}{1-x_{3 \sigma}{ }^{2}}$ zu einem Minimum zu machen. Das Gewicht $\pi_{\sigma}$ wird hiernach von der Lage der Koordinatenachsen abhängig; Herr Bohlin sckwächt aber diese qualitativ unzulässige Abhängigkeit dadurch quan-
Für den von Herrn Kobold behandelten Fall $n=1427$ ergibt sich also

$$
\cos d \cdot \varepsilon(a)=\varepsilon(d)=1 \% 13 \mathrm{I} .
$$

Daß eine Entwicklung nach Potenzen von $m^{2}$, praktisch betrachtet, nicht möglich ist, erkennt man daraus, daß wird.

$$
\mu^{16}=e^{-8 m^{2}}=e^{-5.110}
$$

Die Bedeutung der erlangten Werte der wahrscheinlichen Fehler wird durch die Tatsache, daß nicht alle Fehier $\Delta \omega_{\sigma}$ als zufällige betrachtet werden dürfen, gewil nicht so weit abgeschwächt, daß sie nicht wenigstens als Grenzwerte angesehen werden dürften, die mäßig unterhalb der wirklich begangenen Fehler liegen.

titativ so viel als möglich $a b, d a ß$ er die $x_{3}$-Achse in die Nähe des an die Stelle des Apex tretenden Radianten verlegt. Bei dem Sternschnuppenproblem sind nämlich die Werte aller Größen $v_{\sigma}$ unbeträchtlich, und durch die genannte Wahl

\begin{tabular}{|c|c|c|c|c|c|c|c|c|c|c|c|c|c|}
\hline 1905 & T.m. Padova & $\Delta \alpha$ & $\Delta \delta$ & Cf. & Oss. & \multicolumn{2}{|r|}{$\alpha$ app. } & $\operatorname{og} p \cdot \Delta$ & $\delta$ app & & $\log p \cdot 1$ & Red. ad & \\
\hline 13 & ${ }_{1} x^{\mathrm{h}} 27^{\mathrm{m}} 3^{8^{\mathrm{s}}}$ & $-0^{m} 46: 81$ & $0^{\prime} 44.1$ & 10. & A & $8^{\mathrm{h}}$ & $28^{\mathrm{m}} \mathrm{I}$ & $38_{n}$ & $43^{\prime}$ & & 610 & 5 & \\
\hline I & 105 I 54 & - I $48.9^{8}$ & +230.8 & 8.4 & A & 82 & $27 \quad 9.96$ & $39 n$ & +1845 & & 0 & $07-$ & \\
\hline 16 & 105042 & 01 & +245.0 & 10.5 & A & 82 & 26 & & +1849 & $9 \cdot 3$ & 0.616 & 1 & \\
\hline 23 & I I 29,5 & -329 & -636.8 & 10.5 & A & 8 & $7 \quad 76.4 \mathrm{I}$ & $5 n$ & +19 & 13.0 & $0.59 \mathrm{I}$ & $20-$ & \\
\hline 2 & 72730 & +234.15 & +957.6 & 10.5 & A & 8 & $x 3$ & & $+r g$ & 3 & $0.70 \mathrm{I}$ & $4-$ & \\
\hline 28 & $10 \quad 18 \quad 34$ & +122.06 & +1148.6 & 10.5 & GA & 8 & I 5.3 & & + I9 Io & 42.3 & 0.600 & $4-$ & 4 \\
\hline 31 & $10 \quad 23 \quad 23$ & $-0 \quad 24.42$ & 40.0 & 10.5 & A & 8 & 8 & $.078_{n}$ & +1915 & 23.7 & .593 & $+1.26-11.8$ & \\
\hline
\end{tabular}
der Lage der $x_{3}$-Achse wird nahezu $x_{3}=1, x_{3 \sigma}=v_{\circ}$ und somit unterscheiden sich die Werte der Gewichte $\pi_{\sigma}$ nur wenig von der Einheit. Das Problem, das für $\pi_{\sigma}=I$ dreideutig ist, verwandelt sich durch die Einführung des Näherungspunktes für den Radianten in ein eindeutiges. Bei dem Apexproblem wird das Bohlinsche Verfahren durch den Umstand unzweckmäßig, daß die Größen $v_{\sigma}$ hier zu einem großen Teil beträchtliche Werte besitzen.

\title{
Pianeta 1905 PS
}

osservato all'equatoriale Dembowski $(187 \mathrm{~mm})$ in Padova. 


\begin{tabular}{|c|c|c|c|c|c|c|c|c|c|c|c|c|}
\hline I 905 & T.m. Padova & $\Delta \alpha$ & $\Delta \delta$ & Cf. & Oss. & & $\alpha$ app. & $\log p . A$ & $\delta$ app. & $\log p . \Delta$ & Red. ad 1. app. & * \\
\hline Febb. I & $10^{\mathrm{h}} 28^{\mathrm{m}} 34^{\mathrm{s}}$ & $-I^{m} 30^{s} .26$ & $-6^{\prime}$ I $3^{\prime \prime} 4$ & 10.5 & GA & $8^{h}$ & $7^{\mathrm{m}} 44^{\mathrm{s}} 68$ & $9.004 n$ & $+19^{\circ} 16^{\prime} 50^{\prime \prime} 4$ & 0.591 & $+15.27-11: 7$ & \\
\hline 3 & I0 3723 & $\begin{array}{ll}-0 & 1.73\end{array}$ & $\begin{array}{ll}-6 & 0.9\end{array}$ & 10.5 & GA & 8 & 546.36 & $8.8 \times 9 n$ & $+19 \times 37.3$ & 0.586 & $+1.27-11.7$ & 6 \\
\hline 4 & I0 129 & -0 29.04 & +220.2 & 8.5 & GA & 8 & 450.79 & $9.093 n$ & $\operatorname{tIg} 205^{2.1}$ & 0.592 & $+1.27-11.7$ & \\
\hline 5 & IO 457 & -o $3^{8.42}$ & +243.8 & 10.5 & $\mathrm{GA}$ & 8 & 354.99 & $9.035 \mathrm{n}$ & +1922 & 0.590 & $+1.27-11.7$ & \\
\hline 6 & 104928 & to 18.73 & -6 & 10.5 & GA & 8 & 258.85 & $8.203 n$ & +192316.9 & 0.582 & $+1.27-11.7$ & \\
\hline 9 & 10 $48 \quad 43$ & +230.26 & $-0 \quad 16.4$ & 10.5 & GA & 8 & $\circ \quad 24.65$ & 8.039 & +192618.3 & $0.5^{82}$ & $+1.27-11.6$ & \\
\hline IO & $1037 \quad I$ & +142.65 & +o 37.4 & 10.5 & $\mathrm{GA}$ & 75 & $59 \quad 37.04$ & $7.43 \mathrm{I} \mathrm{n}$ & +192712.2 & $0.58 \mathrm{I}$ & $+1.27-11.5$ & \\
\hline I 1 & 10 $55 \quad 52$ & to 55.92 & +128.0 & 10.5 & A & 75 & $5^{8} \quad 50.30$ & 8.627 & +19282.8 & 0.582 & $+1.26-11.5$ & 20 \\
\hline I 3 & 75026 & -o 25.69 & +250.3 & 10.5 & GA & 75 & $\begin{array}{lll}57 & 28.69\end{array}$ & $9.437 n$ & +192925.1 & $0.4^{8} 4$ & $+1.26-I x .5$ & 10 \\
\hline I 4 & 8 I 7 1 3 & $\begin{array}{ll}-1 & 7.29\end{array}$ & +327.7 & 10.5 & GA & 75 & $5^{6} \quad 47.08$ & $9 \cdot 345 n$ & +19302.5 & 0.612 & $+1.25-11.5$ & 10 \\
\hline
\end{tabular}

Stelle di confronto.

\begin{tabular}{|c|c|c|c|}
\hline * & $\alpha 1905.0$ & $\delta 1905.0$ & Autorità \\
\hline 1 & $8^{\mathrm{h}} 28^{\mathrm{m}} 57^{\mathrm{s}} .87$ & $+18^{\circ} 43^{\prime} 22^{\prime \prime} 8$ & AG. Berlin A. 3398 \\
\hline 2 & $\begin{array}{lll}8 & 24 & 40.55\end{array}$ & +184645.9 & $\gg 3359$ \\
\hline 3 & $\begin{array}{lll}8 & 20 & 44.97\end{array}$ & +1991.6 & $» 3330$ \\
\hline 4 & 8 10 30.48 & $+18595 \cdot 5$ & 》 3257 \\
\hline 5 & $\begin{array}{lll}8 & 9 & 13.67\end{array}$ & +192315.5 & BB.VI + I $9^{\circ} 195^{8}$ \\
\hline
\end{tabular}

Padova, 1905 Febbraio.

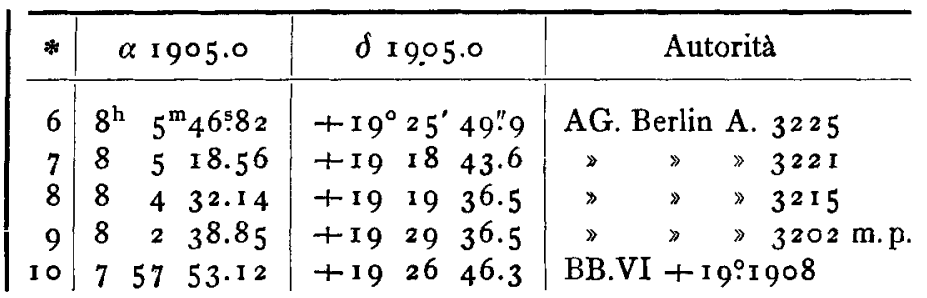

A. Antoniazzi.

Beobachtungen des Kometen 1904 e.

\begin{tabular}{|c|c|c|c|c|c|c|c|c|c|c|c|}
\hline 1905 & M. Ortszeit & $\Delta \alpha$ & $\Delta \delta$ & $\mathrm{Vgl}$. & Gr. & $\alpha$ app. & $|\log p .4|$ & $d$ app. & $\log p .4 \mid$ & Red. ad l. app. & 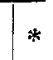 \\
\hline
\end{tabular}

Am Äquatoreal Brunner (0.16 m) der Sternwarte in Lyon von M. F. Guillaume.

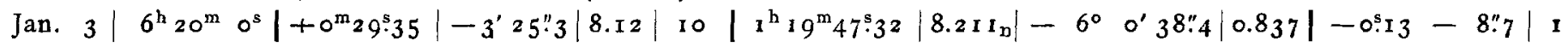
Les images sont diffuses et des nuages gênent par moments; il semble que la comète a un petit noyau.

Am 9-Zöller der Sternwarte in Hamburg von Dr. K. Graff.

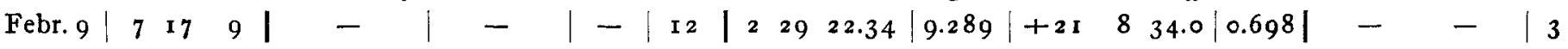

Komet sehr schwach.

Am großen Refraktor der Sternwarte in Straßburg von Dr. E. Fost.

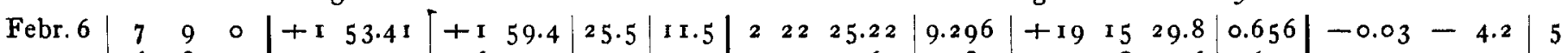

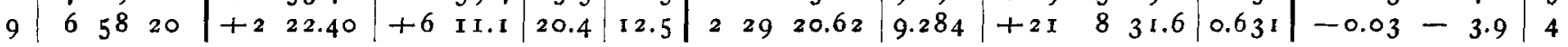

Febr. 6: Komet ohne ausgeprägten Kern. - Febr. 9: Komet im Dunst schwach, höchstens $12^{\frac{\mathrm{m}}{5} 5}$.

Mittlere Örter der Vergleichsterne.

\begin{tabular}{|c|c|c|c|c|c|c|c|}
\hline$*$ & $\alpha 1905.0$ & $\delta 1905.0$ & Autorität & $*$ & $\alpha \mathrm{I} 905.0$ & $\delta \mathrm{I} 905.0$ & Autorität \\
\hline $\mathbf{I}$ & $1^{\mathrm{h}} 19^{\mathrm{m}} 18.10$ & $-5^{\circ} 57^{\prime} \quad 4^{\prime \prime} 4$ & BD. $-6: 2$ & 4 & $2^{\mathrm{h}} 26^{\mathrm{m}} 5^{8.25}$ & $+2 I^{\circ} \quad 2^{\prime} 24^{\prime \prime} 4$ & AG. \\
\hline $\begin{array}{l}2 \\
3\end{array}$ & $\begin{array}{llr}1 & 19 & 3.94 \\
2 & 29 & 11.19\end{array}$ & 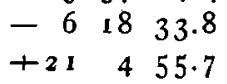 & $\begin{array}{l}\text { AG. Ottakr. } 28 \mathrm{r} \\
9^{\mathrm{m}} \mathrm{r} \text {, Anschl. Hamb. an } 4\end{array}$ & 5 & $2 \quad 20 \quad 31.84$ & +191334.6 & $\begin{array}{c}\text { BD. }+19^{\circ} 35^{6} \text {, Anschl. an } \\
+18^{\circ} 3 \circ 3 \text {, Bonn Vf. } 5\end{array}$ \\
\hline
\end{tabular}

(383) Janina. Korr. der Ephemeride (R. I. Vf. 26 ): Febr. $12+48^{\mathrm{s}}-\mathrm{r}: 9$ Gr. I $2^{\mathrm{m}_{5}}$. $\mathcal{F}$. Palisa.

(512) $[1903 \mathrm{LV}]$. Der Planet hat mit Erlaubnis des Entdeckers von den Turiner Astronomen den Namen Taurinensis erhalten.

Inhalt zu Nr. 3998. P. Harzer. Über die Bestimmung des Apex. 225. - A. Antoniazzi. Pianeta 1905 PS. 237. - Beobachtungen des Kometen J904 e. 239. - Kleine Mitteilungen. 239. 